an important role in the modern epidemiological investigations of tuberculosis in animals at the regional and international level. Bovine tuberculosis represents a significant economic burden to the agriculture of the affected countries. From 2000 to 2015 the disease shows cyclicity in private farms in different regions of Bulgaria. This study is a first molecular investigation of animal tuberculosis in the veterinary medicine in country. The macroscopic and microscopic observation of 35 diagnostic materials from slaughtered cattle, received in the National Reference Laboratory of animal tuberculosis were studied with the three molecular methods: RD4PCR, spoligotyping and MIRU-VNTR. In 27 of the examined lymph nodes we found specific lesions for bovine tuberculosis. The findings were confirmed bacteriologically and by conventional PCR. To differentiate $M$. bovis from other $M$. tuberculosis complex subtypes, we used primers flanking specific deletion (RD4) in the genome of $M$. bovis and obtained the $446 \mathrm{bp}$ DNA product. The spoligotyping subdivided the strains into 3 spoligotypes shared by two to 20 strains. Further molecular investigations of $M$. bovis strains are needed to characterize the genetic diversity and population structure of $M$. bovis strains isolated from cattle in Bulgaria. New information will be added to the global database in the field of molecular epidemiology of the prevalence of $M$. bovis strains in the cattle population in Bulgaria, which will allow comparative analysis with data from the Balkan region and Europe.

Acknowledgements: This work was supported by the grant DN16/12 of the National Science Fund, Bulgaria.

\section{INTERNATIONAL VALIDATION OF ANALYSIS PIPELINES FOR WHOLE GENOME SEQUENCING DATA OF MYCOBACTERIUM TUBERCULOSIS ISOLATES}

D. van Soolingen ${ }^{1}$, R. Jajou ${ }^{1}$, T. Kohl ${ }^{2}$, T. Walker ${ }^{3}$, S. Niemann ${ }^{2}$, H. de Neeling ${ }^{1}$, R. Anthony ${ }^{1}$

${ }^{1}$ National Institute for Public Health and the Environment (RIVM), Bilthoven, The Netherlands; ${ }^{2}$ German Center for Infection Research, Borstel Site, Borstel, Germany; ${ }^{3}$ Nuffield Department of Medicine, University of Oxford, John Radcliffe Hospital, Oxford, United Kingdom

The aim of this multicenter study was to validate and compare different pipelines used for analysis of the Whole Genome Sequencing (WGS) data of Mycobacterium tuberculosis isolates.

All 535 M. tuberculosis isolates of culture positive cases in the Netherlands in 2016, were subjected to WGS, in addition to the routine application of VNTR typing. Transmission suggested on basis of identical VNTR profiles of cases in 2016 was further investigated by municipal health services and 41 epi-links were traced. Fastq.gz files of all 535 samples were analysed in four different WGS pipelines to facilitate international comparison: 1) SNP-based method at the RIVM/Bilthoven/ The Netherlands; 2) SNP-based method at Oxford University/UK; 3) SNP-based method and 4) cgMLST at Borstel/Germany.

In all pipelines, shorter than $12 \mathrm{SNP}$ distances between the 41 epi-linked cases was observed. One epi-linked pair revealed a higher genetic distance of 27 SNPs in the Bilthoven pipeline, due to poor sequence quality resulting in low coverage. In general, the genetic distances between isolates of the epi-linked cases were smaller in the Oxford and Borstel pipelines ( $0-3$ SNPs), than in the Bilthoven pipeline (1-11 SNPs). All pipelines clustered roughly the same cases, more isolates without identified epi-links were clustered in the Oxford $(n=34)$ and both Borstel pipelines $(n=32$ in the SNP pipeline and $n=39$ in the cgMLST) than the Bilthoven pipeline $(n=29)$.

Also, some cases not clustered by VNTR were clustered by WGS. Patient characteristics revealed that in some of these pairs of cases an epi-link, missed by VNTR typing, was likely.

Several differences were observed among the pipelines with regard to the version of reference genome used, software used for mapping and SNP calling, (repetitive) regions excluded in the analysis, the minimum number of reads to support SNPs, and the minimum allele frequencies. The RIVM pipeline was adapted in the light of these results to function more in line with other international laboratories pipelines, facilitating the comparability of results.

International standardization on all these variables is necessary, and subsequently on the SNP cut-off to be applied to WGS clustering, to allow international-laboratory comparison of WGS data and reliable investigation of cross-border transmission.

6.58

doi: 10.15789/2220-7619-2018-4-6.58

\section{RNA-BASED DRUG SUSCEPTIBILITY TESTING OF MYCOBACTERIUM TUBERCULOSIS}

A. Van den Bossche ${ }^{1}$, R. Bhattacharyya ${ }^{2}$, J.Y. Coppee ${ }^{3}$,

L. Rigouts ${ }^{4}$, A. Baulard ${ }^{5}$, A. Vodolazkaia ${ }^{6}$, D. Hung ${ }^{2}$,

V. Mathys ${ }^{1}$, P.J. Ceyssens ${ }^{1}$

${ }^{1}$ Division of Bacterial Diseases, Sciensano, Brussels, Belgium; ${ }^{2}$ Harvard Medical School, Boston, United States; ${ }^{3}$ Institut Pasteur, Paris, France; ${ }^{4}$ Institute of Tropical Medicine, Antwerp, Belgium; ${ }^{5}$ Center for Infection and Immunity of Lille, Institut Pasteur de Lille, France; ${ }^{6}$ Laboratory of Medical Microbiology, Sciensano, Brussels, Belgium

Multidrug resistant tuberculosis (MDR-TB) is one of the major WHO health concerns. One of the challenges that hampers the effective response to MDR-TB is the long turnaround time of phenotypic Drug Susceptibility Testing (DST) of Mycobacterium tuberculosis. To counter this, new fast and sensitive DNA-based methods were successfully introduced over the last years. However, these (a) are based on the knowledge on resistance mutations, (b) do not distinguish living from dead cells, (c) ignore all intrinsic resistance mechanisms, and (d) ignore the influence of compensatory mutations.

We introduce a next-generation diagnostic test based on quantification of drug-specific RNA biomarkers. The basic principle is that a brief antibiotic exposure triggers specific transcriptional responses in susceptible, but not in resistant, microbes within a few hours. This has the advantage that long culture-dependent steps are avoided, yet the resistance phenotype is detected independent of the specific cause of resistance.

First, the global transcriptional response of two M. tuberculosis strains to 10 anti-TB drugs was determined using RNAtaq-Seq. A set of highly responsive genes was selected for each drug and RNA-targeting probes were designed.

Next, the RNA-based DST was developed in 96 well format. In short, $200 \mu \mathrm{l}$ of a positively flagged MGIT ${ }^{\mathrm{TM}}$ (BD) culture is spiked with a drug, while a replicate is incubated in absence of the drug. Multiplex mRNA quantification is performed directly on crude cell lysates using a combination of the bead-based $\mathrm{MagPix}^{\mathrm{TM}}$ (Luminex) and Quantigene ${ }^{\mathrm{TM}}$ Plex (Thermo Fisher) technology. 
The normalized expression levels are combined to one numeric value which determines the drug susceptibility of the investigated strain

We successfully developed 8 primary sets of RNA biomarkers for ten $1^{\text {st }}$-line, $2^{\text {nd }}$-line and new drugs. Taking isoniazid as proof of principle, we present a biomarker set of 5 responsive genes and 3 normalizing genes, which enables to distinguish susceptible, low- and high resistant TB strains after 6 hours incubation. Next, preliminary results demonstrate that the biomarker sets can successfully discriminate between susceptible and resistance strains for the selected drugs.

We present a robust, RNA-based DST without the need for RNA extraction. The assay was proven to be efficient for isoniazid. With a total of 8 biomarker sets under optimization, the drug resistance profile of up to 14 drugs can be determined.

\subsection{9} doi: 10.15789/2220-7619-2018-4-6.59

\section{POPULATION STRUCTURE OF MYCOBACTERIUM TUBERCULOSIS IN RUSSIAN REGIONS BORDERING EU COUNTRIES}

A. Vyazovaya ${ }^{1}$, N. Solovieva ${ }^{2}$, A. Gerasimova ${ }^{1}$

G. Akhmedova ${ }^{3}$, E. Turkin ${ }^{3}$, T. Sunchalina ${ }^{4}$, R. Tarashkevich ${ }^{5}$, S. Bogatin ${ }^{5}$, N. Gavrilova ${ }^{6}$, A. Bychkova ${ }^{6}$, E. Anikieva ${ }^{6}$, V. Zhuravlev ${ }^{2}$, O. Narvskaya ${ }^{1,2}$, I. Mokrousov ${ }^{1}$

${ }^{1}$ St. Petersburg Pasteur Institute, St. Petersburg, Russia; ${ }^{2}$ Research Institute of Phthisiopulmonology, St. Petersburg, Russia; ${ }^{3}$ Regional Tuberculosis Dispensary, Kaliningrad, Russia $:{ }^{4}$ Republican

Tuberculosis Dispensary, Petrozavodsk, Karelia, Russia; ${ }^{5}$ Regional Tuberculosis Dispensary, Pskov, Russia; ${ }^{6}$ Regional Tuberculosis

Dispensary, Murmansk, Russia

Population structure of Mycobacterium tuberculosis in Russia is characterized by predominance of the Beijing genotype strains. A high burden of multidrug-resistant (MDR) tuberculosis in the northwestern Russia may have a negative impact on TB control programs in the neighboring countries of the European Union.

We aimed to assess the population structure of M. tuberculosis in northwestern Russian regions (Karelia, Kaliningrad, Murmansk, Pskov) bordering countries of the European Union (Finland, Norway, Estonia, Latvia, Lithuania and Poland).

A total of $304 M$. tuberculosis isolates (2013-2017) from newly diagnosed TB patients in geographically distant regions of northwestern Russia were studied: Republic of Karelia $(n=78)$, Pskov $(n=89)$, Kaliningrad $(n=73)$ and Murmansk provinces $(n=66)$. The Beijing genotype family and its particular clusters B0/W148 and 94-32 were detected by analysis of specific markers in $\mathrm{dnaA}$ dnaN::IS6110, Rv2664-Rv2665::IS6110 and sigE98, respectively. Non-Beijing isolates were subjected to spoligotyping followed by comparison to the SITVIT_WEB.

These 4 Russian regions differed to some extent in the prevalence of the Beijing genotype: from to $55.1 \%$ in Karelia, $44.9 \%$ in Pskov, $63.0 \%$ in Kaliningard and $51.5 \%$ in Murmansk. Beijing B0/W148-cluster was identified in $17.9 ; 6.7 ; 19.2$ and $10.6 \%$ isolates, respectively. The prevalence of the Beijing 94-32-cluster did not differ: $28.2 ; 29.2 ; 28.8$ and $30.3 \%$, respectively. In Pskov and Murmansk, most strains were drug susceptible (62.9 and $62.1 \%$ ), while $17.9 \%$ and $25.8 \%$ were MDR, respectively. In contrast, MDR strains prevailed in Karelia and Kaliningard - 51.3 and $43.8 \%$, while 41.0 and $36.9 \%$ strains were susceptible. In total, for four regions together, 90.2\% (37/41) strains of the Beijing B0/W148-cluster were MDR. Beijing 94-32-cluster strains showed much lower rate of MDR - 34.8\%, (31/89) ( $\mathrm{P}<0.0001)$, while $41.6 \%$ (37/89) were susceptible. Among non-Beijing M. tuberculosis the largest families were T (14.1 and $15.1 \%)$ in Karelia and Kaliningard, LAM (23.6\%) in Pskov, and Ural (19.7\%) in Murmansk.

M. tuberculosis population in the northwestern Russian-EU border provinces is marked by predominance of the major clonal complexes of the Beijing genotype (B0/W148 и 94-32). However, these clones significantly differed in the proportion of MDR. A circulation of the MDR-associated isolates of the Beijing B0/W148-cluster presents the major concern for local and national TB control programs in the Russian regions and likely adverse impact on the neighboring EU countries.

Funding: Russian Science Foundation grant 14-14-00292.

6.60 doi: 10.15789/2220-7619-2018-4-6.60

\section{DRUG RESISTANCE IN MYCOBACTERIUM} TUBERCULOSIS: FROM PHENOTYPIC MIC-ANALYSIS TO WGS FOR ROUTINE DRUG SUSCEPTIBILITY TESTING

\section{J. Werngren}

Supranational Reference Laboratory for Tuberculosis and Public Health Agency of Sweden, Department of Microbiology, Unit of Laboratory Surveillance of Bacterial Pathogens, Solna, Sweden

The phenotypic methods used for drug susceptibility testing (DST) of Mycobacterium tuberculosis generally take weeks or months and require culture and manipulation of large numbers of highly infectious bacilli. Drug resistance in $M$. tuberculosis is almost exclusively a consequence of genomic mutations and so molecular determination of resistance offers a rapid, potentially cost effective, and safer alternative.

In 2016, the SRL in Stockholm introduced whole genome sequencing (WGS) for the molecular typing of clinical isolates of M. tuberculosis. At the same time, the WGS analysis made information of various putative drug resistance-related mutations available, which was utilized to complement the routine phenotypic DST. Since 2016, the WGS information on resistance to all clinically relevant drugs is analysed and routinely reported together with the extended phenotypic DST results for all the Swedish multidrug resistant (MDR) isolates.

Retrospectively, we have performed an observational study to compare the WGS data to the phenotypic DST to predict the resistance phenotype from the genetic sequence. The study included 877 clinical TB isolates (only one sample per patient was included) received by the Public Health Agency of Sweden between 01.01.2016 and 31.12.2017. The analysis of the isolates' resistance profiles for the first and second line drugs, obtained from the WGS and Bactec MGIT 960, is ongoing.

Simultaneously, we are evaluating the microbroth dilution technique used for the MIC determination of 150 consecutive (MDR) M. tuberculosis together with the corresponding WGS analysis as a part of a collaboration with the CRyPTIC (Comprehensive Resistance Prediction for Tuberculosis: an International Consortium) network. The MIC determinations of drug susceptible and resistant $M$. tuberculosis isolates are necessary in the work to establish correct critical test concentrations for phenotypic DST as well as to identify the minimal, moderate and high confidence mutations. Such coupled analysis will more accurately define clinical drug resistance and may possibly constitute the DST algorithm of choice in an increasing number of laboratories. 